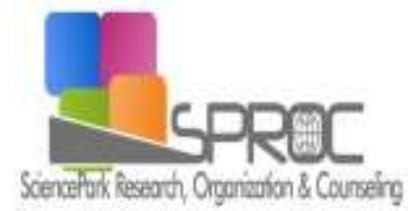

\title{
National energy strategies of Germany and Turkey
}

Anil Berkin *

Suggested Citation:

7

Abstract 
1. Introduction and Background 
2. System Descriptions

3. Critical Analysis of Literature

3.1. General Energy Policy

\subsubsection{Germany}


Germany can easily replace its nuclear capacity on the phaseout schedule Replacing nuclear plants with reserves, new renewables, gas, and demand-side management (DSM)

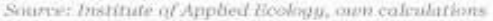

$20.9 \mathrm{GW}$ of nuclear capacity

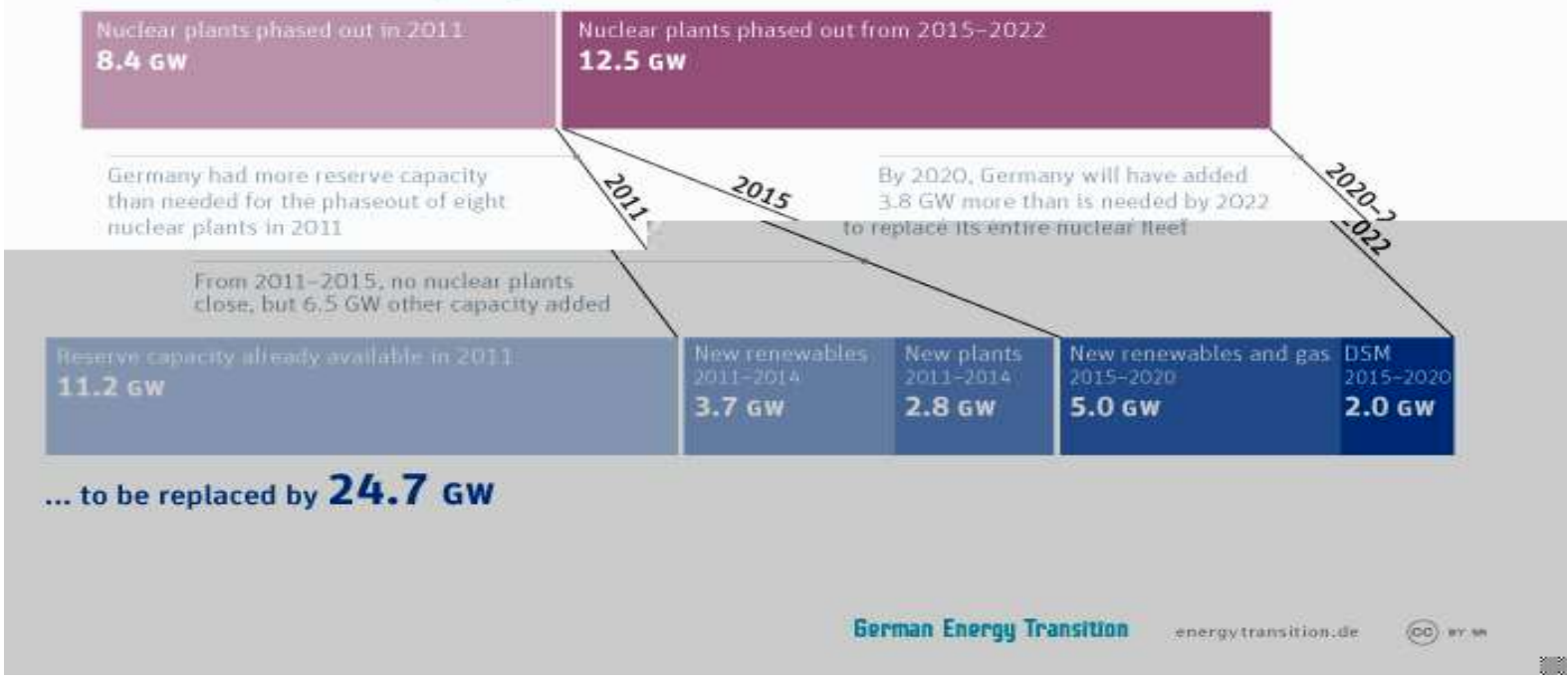

Source: energytransition.de 
Total primary energy supply, 1973-2011

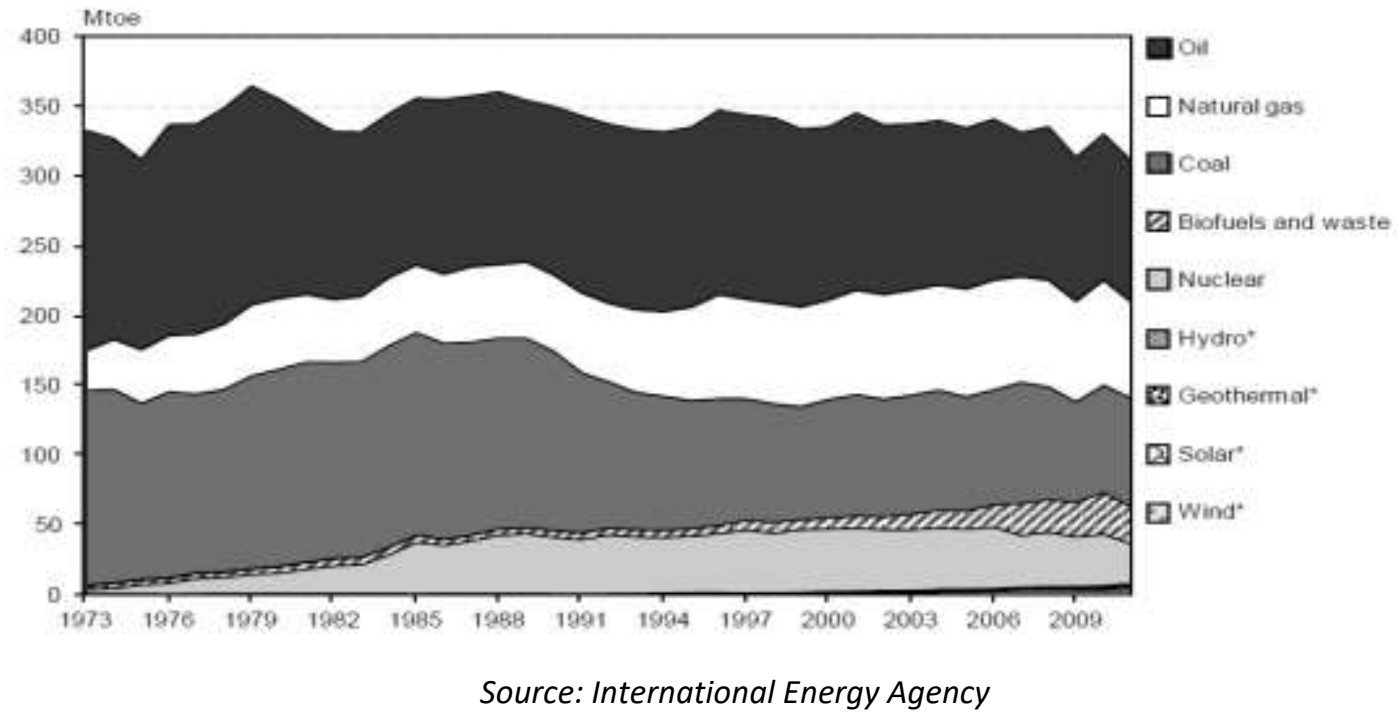

3.1.2. Turkey 
Total Primary Energy Supply, 1973 to 2008

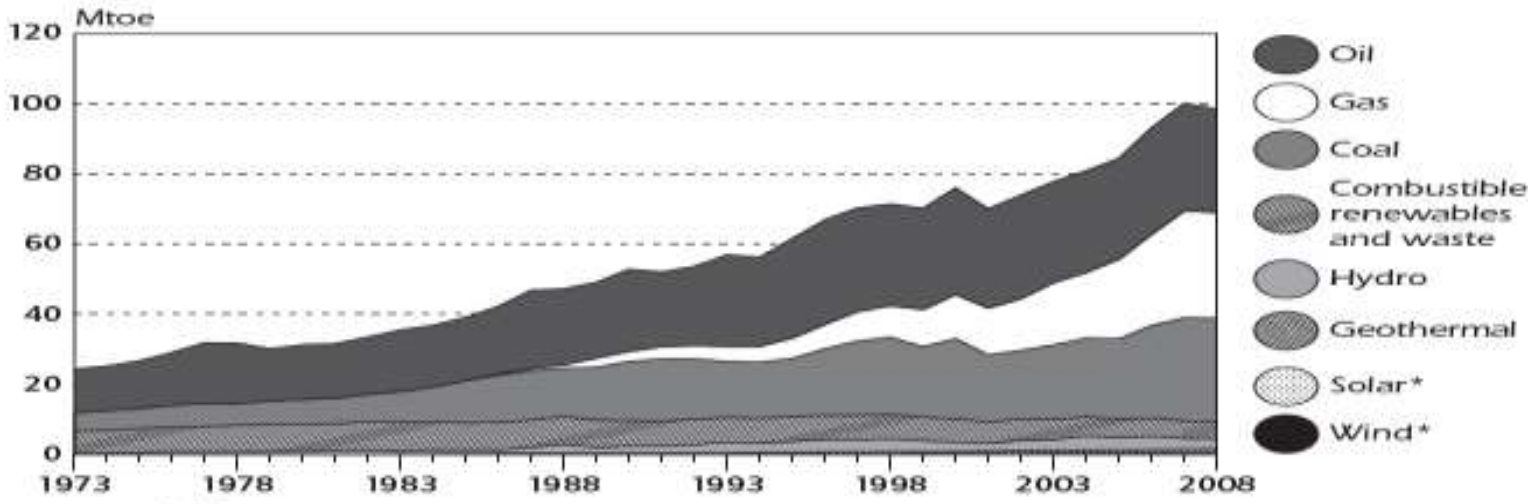

* negligible.

Source: Energy Balances of OECD Countries, IEA/OECD Paris, 2009

\subsection{Energy Production and Consumption}

\subsubsection{Germany}

Primary Energy Production

in 2014 (Germany, Mtoe)

\begin{tabular}{lr} 
Energy & Mtoe \\
\hline Vind Elec & 5,0 \\
Oil & 8,1 \\
$\square$ Nuctear & 23,9 \\
Gas & 9,3 \\
Other Renevwables & 12,8
\end{tabular}

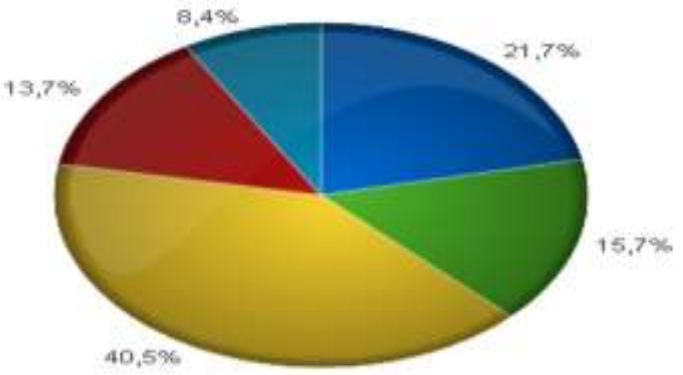

Total $=59,1$ Mtoe

mes suirr phoject daya romtaL. 


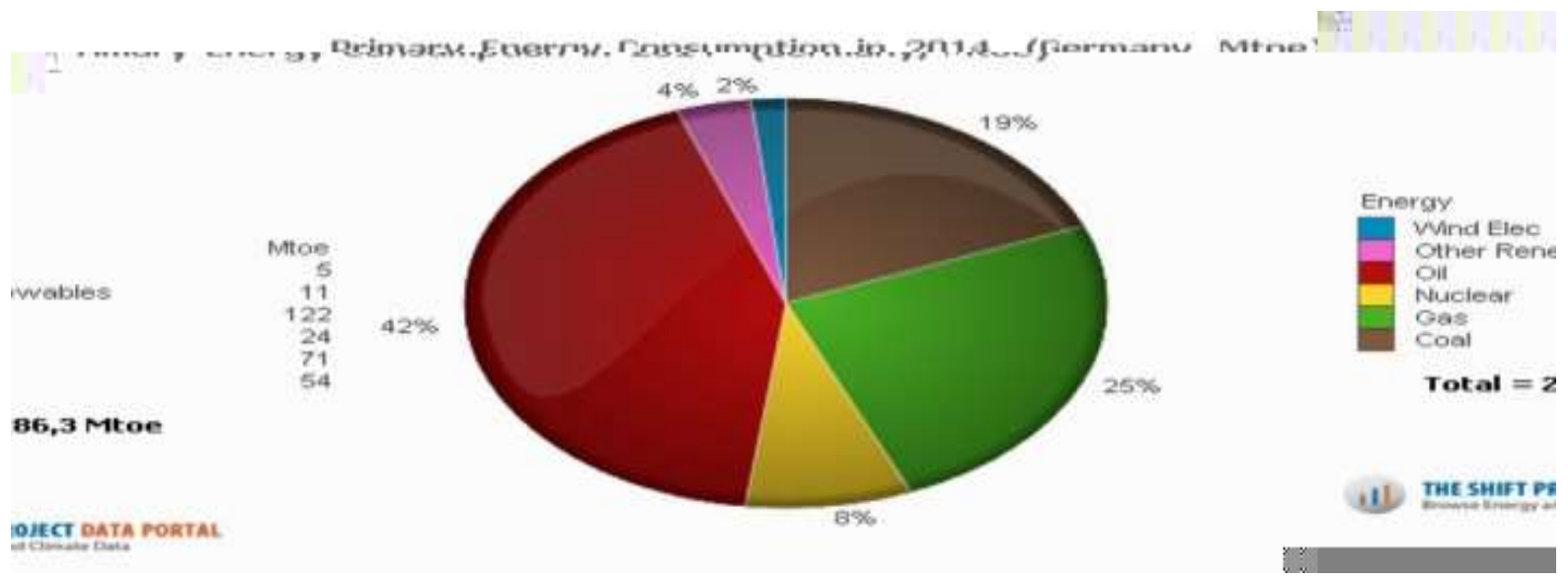

Source: The Shift Project Data Portal 
Figure 18. Crude oil imports by country of origin, 2011

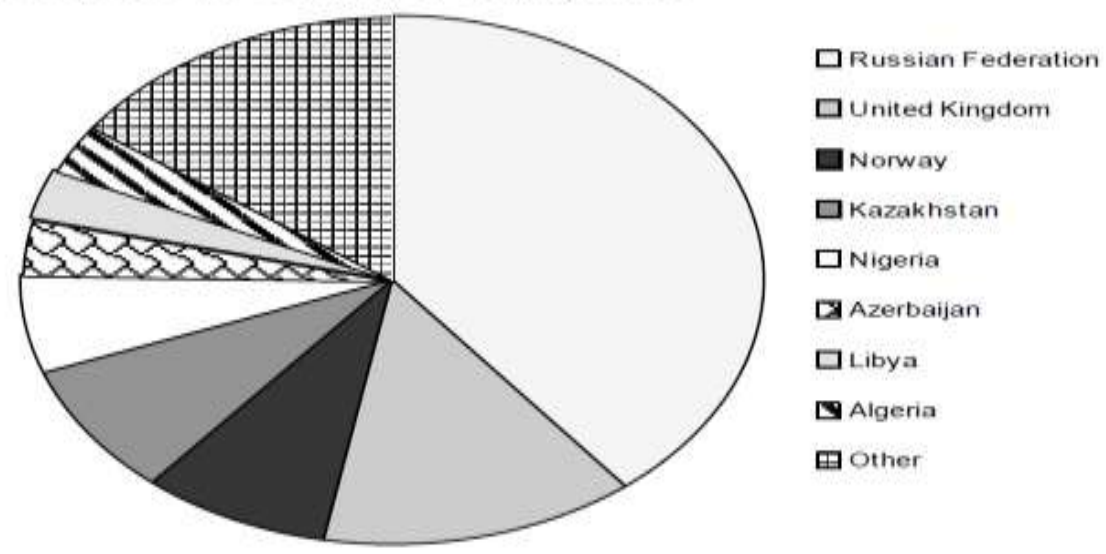

Source: Oll, Gas, Coal and Electricity Quarterly Statistics, Second Quarter 2012, IEAVOECD 2012.

\subsubsection{Turkey}

Primary Energy Production in 2014 (Turkey, Mtoe)

Energy

Vyind Eloc

all

Hyctroelectricity

Coal

Other Renewables

Total $=24,5$ Mtoe

THE SHET PaOJeCr DATA Fomral.

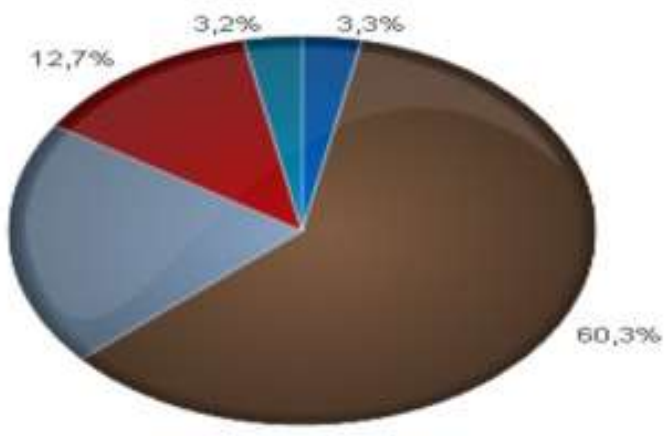

Source: The Shift Project Data Portal 
Primary Energy Consumption in 2014 (Turkey, Mtoe)

Energy

Wind Elec

Other Renewables

Oil

Hydroelectricity

Gas

coal

Total $=108,2$ Mtoe

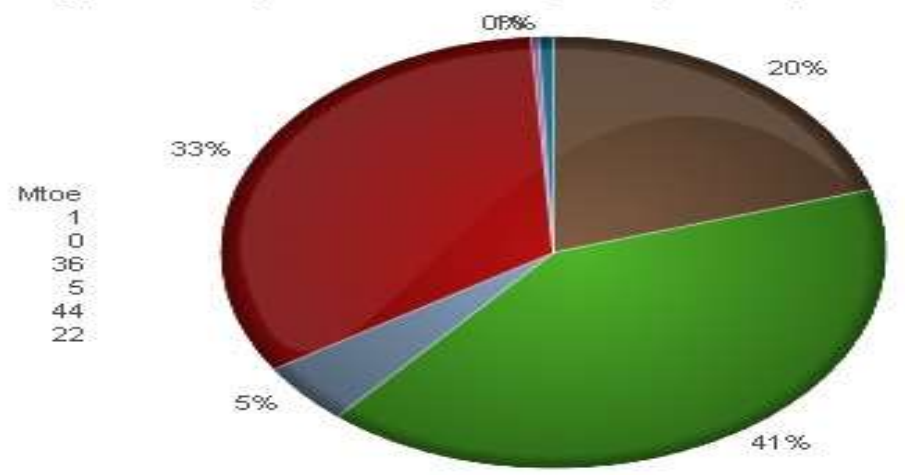

THE SHIFT PROJECT DATA PORTAL.

Source: The Shift Project Data Portal 


\section{Figure 2. Turkey petroleum and other liquids consumption and production}

thousand barrels per day

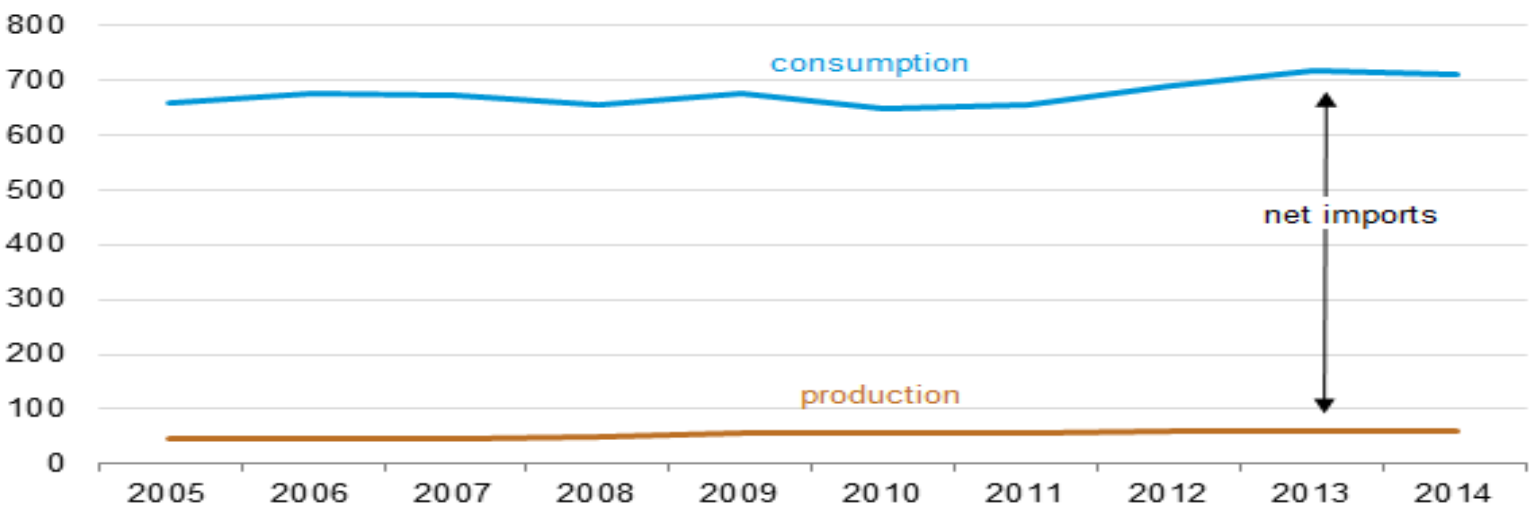

ia Note: 2014 consumption is a preliminary estimate.

Cia Source: U.S. Energy Information Administration

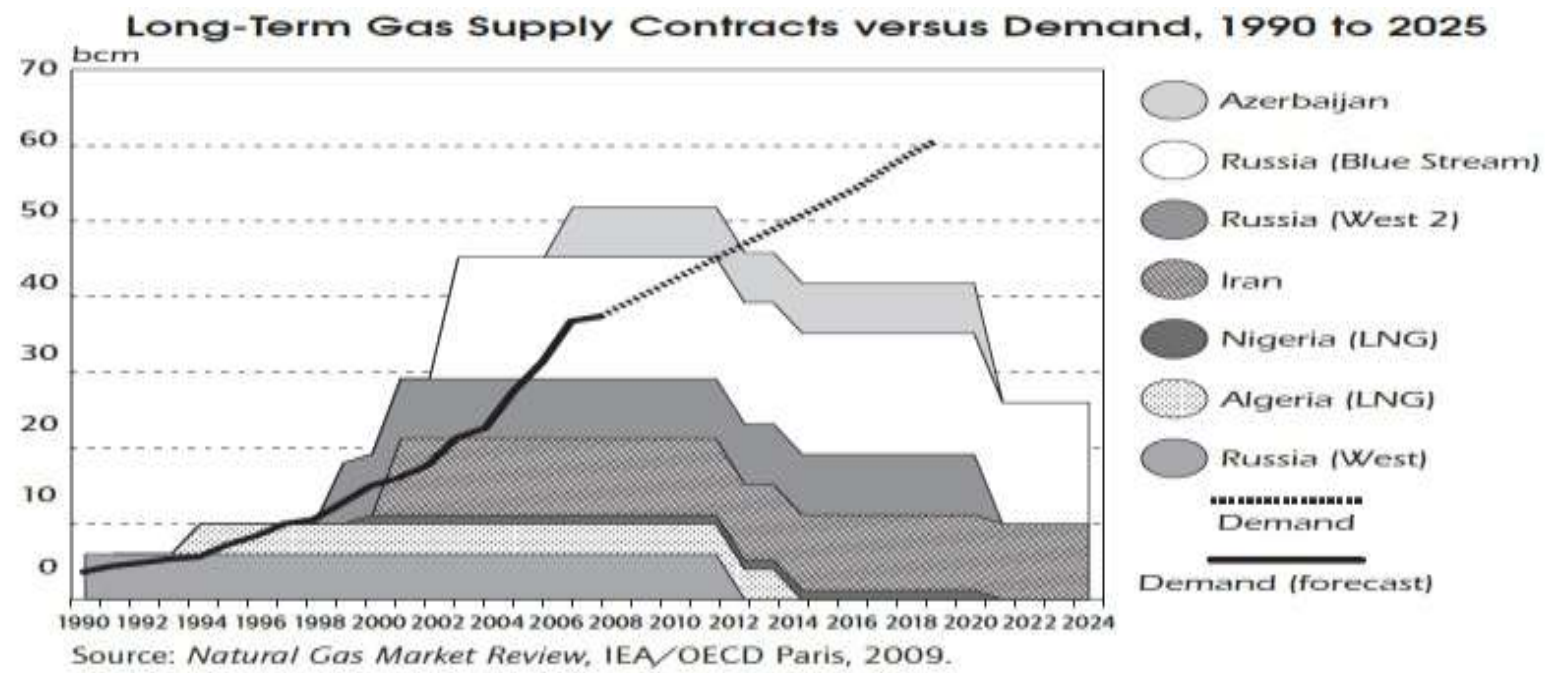


4. Conclusion 


\section{References}

From past to present relations between Germany and Turkey.

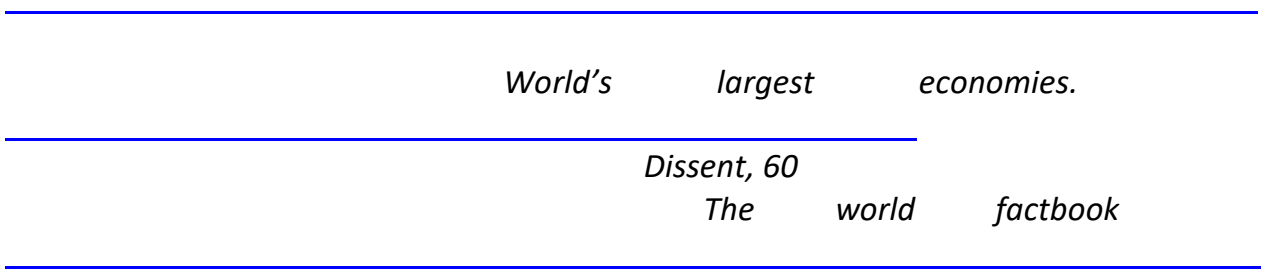

Country factsheet

Germany. 